plaques. Growth factors and inflammatory cytokines are potential serum biomarkers to identify patients at risk of stroke.

Materials and methods Immunohistochemistry and quantitative PCR (Q-PCR) were used to establish localisation and expression of angiogenic growth factors within carotid endarterectomy specimens from symptomatic and asymptomatic patients. Stable or unstable microvessels were distinguished by CD31 and CD105 staining. Systemic levels of circulating angiogenic growth factors and inflammatory cytokines were measured in venous blood using BioPlex arrays.

Results Hepatocyte growth factor (HGF) and its receptor c-Met were detected in CD31-positive endothelia, and $\boldsymbol{\alpha}$-SMA-positive cells, respectively. Q-PCR demonstrated upregulation of the angiogenic factors CD105, HGF $(p<0.001)$ and $c-$ Met $(p=0.011)$ in symptomatic versus asymptomatic plaques. A significantly greater neovessel density was detected in symptomatic plaques $(p=0.042)$, associated with elevated expression of HGF and c-Met. Suspension arrays demonstrated elevated HGF $(p=0.002)$ and decreased plateletderived growth factor (PDGF; $\mathrm{p}=0.036$ ) serum levels in symptomatic versus asymptomatic patients. Twenty-seven cytokines were examined; seven endarterectomy patients demonstrated significantly increased levels in comparison with controls. No differences were observed between preoperative and postoperative serum.

Discussion Plaque instability may be mediated by HGF-induced formation of microvessels, and decreased PDGF. We will investigate the effects of inflammatory cytokines with a view to comparing symptomatic versus asymptomatic patients. Targeting surgery to those who will benefit would eliminate unnecessary risk.

\section{BAS/ PARTIAL RECONSTRUCTION OF MYOCARDIAL METABOLIC BSCR3 PATHWAYS FOLLOWING ANALYSIS OF PERIPHERAL SERUM USING METABOLOMICS IN EARLY CARDIAC ISCHAEMIA}

\author{
doi:10.1136/hrt.2010.205781.14
}

${ }^{1} \mathrm{M}$ A Mamas, ${ }^{2} \mathrm{~W}$ B Dunn, ${ }^{2} \mathrm{D}$ Broadhurst, ${ }^{1} \mathrm{~S}$ Chacko, ${ }^{3} \mathrm{M}$ Brown, ${ }^{1} \mathrm{M}$ El-Omar, ${ }^{1} \mathrm{~F}$ FathOrdoubadi, ${ }^{2} \mathrm{R}$ Goodacre, ${ }^{2,3} \mathrm{D}$ B Kell, 'L Neyses. ${ }^{1}$ Biomedical Research Centre, Central Manchester NHS Foundation Trust, UK; ${ }^{2}$ Manchester Centre for Integrative Systems Biology, University of Manchester, UK; ${ }^{3}$ Bioanalytical Sciences Group, Manchester Interdisciplinary Biocentre, University of Manchester, UK

Cardiac metabolism and cardiac function are inextricably linked, with changes in cardiac metabolism during cardiac ischaemia contributing to the development of cardiac arrhythmias. Using metabolomics, we aim to identify metabolite changes occurring during cardiac ischaemia through analysis of peripheral serum to reconstruct myocardial metabolic pathways that contribute to the development of cardiac arrhythmias. Peripheral venous samples from 25 patients (and 20 patients in a validation cohort) were analysed in an untargeted fashion using LC-MS following cardiac ischaemia induced by transient coronary artery occlusion during PCI at baseline, 1 and 5 min. Following validation, 99 and 126 metabolite peaks were significantly different at $1 \mathrm{~min}$ and $5 \mathrm{~min}$ after coronary occlusion compared with baseline $(\mathrm{p}<0.05)$. Predominantly metabolic pathways involving lipids were perturbed with changes in diacylglycerols (DG), lysophophatidylcholines (LPC), phosphatidylcholine (PC) and free fatty acids (FFA). Myocardial metabolic pathways involving the synthesis of PC from DG and their subsequent breakdown by phospholipase A2 into LPC and FFA such as arachidonic acid (AA) stimulating the oxidation of adrenaline to form the arrhythmogenic metabolite adrenochrome were reconstructed. We are able to reconstruct metabolic pathways involving lipid metabolism within the myocardium during cardiac ischaemia through analysis of the peripheral serum using metabolomics. Our unbiased approach has identified metabolic pathways involved in the production and release of metabolites with pro-arrhythmic properties (AA, LPC and adrenochrome) and metabolites with anti- arrhythmic properties (omega-3 fatty acids: eicosapentaenoic and docosahexanoic acid). This suggests that arrhythmogenesis may be a delicate balance between the endogenous formation of proarrhythmic and anti-arrhythmic metabolites.

\section{BAS/ THE ROLE OF RECEPTOR ACTIVATOR OF NUCLEAR FACTOR BSCR4 K-B LIGAND AND ITS DECOY RECEPTOR, OSTEOPROTEGERIN IN VASCULAR CALCIFICATION}

doi:10.1136/hrt.2010.205781.15

${ }^{1} \mathrm{~A}$ S Williams, ${ }^{1,2} \mathrm{~A}$ Ndip, ${ }^{2} \mathrm{E}$ Jude, ${ }^{2} \mathrm{~A} J \mathrm{M}$ Boulton, ${ }^{1} \mathrm{M}$ Y Alexander. ${ }^{1}$ Cardiovascular Research Group, Manchester Diabetes Centre, Faculty of Medicine \& Human Science, School of Biomedicine, University of Manchester, UK; ${ }^{2}$ Manchester Royal Infirmary, University Department of Medicine, Oxford Road, Manchester, UK

Charcot neuroarthropathy $(\mathrm{CN})$ is characterised by pathological foot fractures and osteopenia in patients with diabetes, often resulting in debilitating deformity. Paradoxically, these patients show evidence of medial vascular calcification. Recently, accentuated signalling of the receptor activator of nuclear factor $\kappa-B$ ligand (RANKL) and its decoy receptor, osteoprotegerin (OPG) have been implicated in the development of diabetic $\mathrm{CN}$. This study aims to investigate the role of RANKL and OPG signalling in vascular calcification in patients with diabetes and $\mathrm{CN}$, compared with healthy controls. RANKL and OPG serum levels were measured using ELISA in 12 patients with CN, 10 diabetic patients and five healthy controls. Serum RANKL and OPG levels were elevated in acute $\mathrm{CN}$ and in diabetic patients compared with healthy controls $(p<0.05)$. Immunohistochemistry identifies upregulation of RANKL in calcified tibial arterial sections versus non-calcified controls. Human vascular smooth muscle cells (hVSMC) were grown in osteogenic conditions, as our in vitro model of calcification. When hVSMCs were treated with serum from patients with diabetes and $\mathrm{CN}$, we demonstrated (i) accelerated mineralisation of hVSMC, confirmed by Alizarin red staining, and elevated alkaline phosphatase activity compared with control cells and (ii) reduced mineralisation when co-incubated with OPG. These findings demonstrate that RANKL/OPG signalling is modulated in diabetic and $\mathrm{CN}$ patients. Furthermore, serum from these patients accelerates vascular calcification in vitro, an effect attenuated by OPG treatment. These are the first human data implicating RANKL/OPG in diabetic vascular calcification and suggest that OPG/anti-RANKL therapy may be a potential target in combating disease progression.

\section{BAS/ TILRR POTENTIATES INTERLEUKIN-1-INDUCED ANTI- BSCR5 APOPTOSIS}

doi:10.1136/hrt.2010.205781.16

Gemma Montagut Pino, Xiao Zhang, Eva E Qwarnstrom. Cell Biology, Vascular Science, University of Sheffield, UK

The pathogenesis of atherosclerosis is determined, in part, by inflammatory responses, induced through members of the Toll-like and interleukin (IL)-1 receptor family and controlled by NF- $\kappa B$. We have identified a novel IL-1RI co-receptor, TILRR, which enhances the IL-1-induced activation of NF- $\mathrm{KB}$ by increasing receptor-expression enhanced recruitment of the MyD88 adaptor during activation. ${ }^{1}$ Here we investigate the role of TILRR on the anti-apoptotic effects controlled by NF- $\kappa B$. The results showed that TILRR reduces caspase3 activity and enhances IL-1-induced phosphorylation of AKT. Alanine scanning mutagenesis of the IL-1 receptor TIR domain demonstrated that TILRR amplifies inflammatory responses through the membrane proximal part of the cytoplasmic portion, the so-called box 1 , while the anti-apoptotic response is regulated through the central portion of the TIR domain, the so-called box 2. Similarly, 